\title{
A DIFFICULTY WITH THE ADDRESS MODELS OF PRODUCT DIFFERENTIATION*
}

\section{Martin Peitz**}

\author{
WP-AD 98-21
}

Correspondence to:

Martin Peitz. University of Alicante. Facultad de Ciencias Económicas.

Dpto. Fundamentos del Análisis Económico. Ctra. San Vicente del Raspeig, s/n. 03071 ALICANTE-SPAIN

E-mail: peitz@merlin.fae.ua.es

Editor: Instituto Valenciano de Investigaciones Económicas, S.A.

First Edition September 1998

ISBN: 84-482-1865-5

Depósito Legal: V-2884-1998

IVIE working-papers offer in advance the results of economic research under way in order to encourage a discussion process before sending them to scientific journals for their final publication.

* I like to thank Egbert Dierker for helpful discussions and an anonymous referee for helpful comments. Financial support from Instituto Valenciano de Investigaciones Económicas (IVIE) and EU Human Capital and Mobility Program is gratefully acknowledged.

** University of Alicante. 


\title{
A DIFFICULTY WITH THE ADDRESS MODELS OF PRODUCT DIFFERENTIATION
}

\section{Martin Peitz}

\begin{abstract}
In models of product differentiation and location models it is implicitly assumed that consumers can afford to buy the differentiated goods in the market. I show that with income heterogeneity there are severe existence problems of a price equilibrium in models of horizontal product differentiation with unit demand because some consumers are income-constrained. The result generalizes to other models of product differentiation, search, and switching costs. I present an alternative specification of variable individual demand in which this kind of existence problem cannot arise.
\end{abstract}

JEL-Classification: D43, L13

Keywords: Product Differentiation, Unit Demand, Heterogeneous Income, Discrete Choice 


\section{Introduction}

The literature on product differentiation is dominated by one-dimensional models of unit demand, i.e. the characteristics space is one-dimensional and consumers buy one unit of the product which comes in different variants. Starting with a so-called indirect utility function no formal distinction is made between a location model and a model of horizontal product differentiation.

In this note I analyze models of horizontal differentiation where the so-called transportation cost is a disutility due to the difference between the actual variant of the product and the consumer's ideal variant. I derive individual demand functions from utility maximization (section 2). Section 3 studies models of horizontal product differentiation with heterogeneous income. Models of product differentiation suffer from the nonexistence of symmetric price equilibria when for some consumers the willingness to pay exceeds the income (or capacity to pay) and income is sufficiently heterogeneous (Proposition 1). The literature does not address this issue. Proposition 2 shows that in the Hotelling model with quadratic transportation costs also asymmetric price equilibria in pure strategies fail to exist for some locations of the goods in the characteristics space, a result which seems surprising in the light of the literature (given the specification I have chosen). ${ }^{1}$

It should be pointed out that Caplin and Nalebuff (1991) make the incorrect claim that their approach allows for heterogeneous income and that their derived reservation price rule is concave. The Hotelling model with quadratic transportation costs is a special case of their model. The results in their section 8.1 are not correct.

Section 4 contains an alternative specification with variable individual demand in which the non-existence problem of the previous section does not arise. Section 5 concludes with remarks on the generality of the result. In particular, I point out that existence problems also arise in other models of product differentiation (vertical differentiation, quasi-linear preferences, random utility models), and in models of search and switching costs.

\footnotetext{
${ }^{1}$ Only in Peitz (1996) a possibly binding capacity to pay has been considered (in the circle model). The issue of a binding capacity to pay enforcing zero consumption of the differentiated goods also arises in models of vertical product differentiation (see concluding remarks). There the problem has been avoided by explicitly assuming that income is sufficiently high (Mussa and Rosen, 1978, and Peitz, 1995).
} 


\section{Consumer Behavior}

I demonstrate the importance of the budget constraint in discrete choice models when income is heterogeneous by a simple example of a model in the spirit of Hotelling (1929) and Salop (1979). With $\omega$ I denote the type of consumer which is a number denoting his location or ideal point, $\omega \in \Omega \in\left\{[\underline{\omega}, \bar{\omega}], C_{k}\right\} . C_{k}$ denotes the circle with circumference $k$. A consumer is in addition described by his income $y$ which defines his capacity to pay for a unit of any of the goods $i=1, \ldots, n$ available in the market. $l_{i}$ is the location of good $i, l_{i} \in L \in\left\{[\underline{l}, \bar{l}], C_{k}\right\}$ where $L$ is the product space which is either an interval or the circle with circumference $k$. The transportation cost function $t: \Re_{+} \rightarrow \Re_{+}$depends on the distance between consumer and good. It is assumed to be convex and $t(0)=0$. In a model of product differentiation the transportation cost describes the disutility which is due to the distance between the location of good $i$ in the product space and the ideal point of a consumer. $z$ denotes a positive constant which will be interpreted below. In models of horizontal product differentiation or location models consumers are described by an evaluation function (often a positive constant is added)

$$
\begin{aligned}
v^{\omega, y}(p) & =\max \left\{0,\left(v_{i}^{\omega, y}\left(p_{i}\right)\right)_{i}\right\} \text { with } \\
v_{i}^{\omega, y}\left(p_{i}\right) & =z-t\left(\left|\omega-l_{i}\right|\right)-p_{i}
\end{aligned}
$$

In the literature (see e.g. chapters 6 and 8 in Anderson, de Palma and Thisse, 1992, or section 2.4 in Beath and Katsoulacos, 1991) the functions $v^{\omega, y}$ are called indirect utility functions. As I will show, $v^{\omega, y}$ cannot always be used in models of product differentiation. In contrast, location models do not suffer from this problem because the transportation cost has to be paid out of the budget.

Below I construct conditional direct utility functions. Consumers derive utility from one unit of one of the differentiated goods and the composite commodity 0 which is perfectly divisible. Consumers are nonsatiated in the composite commodity and therefore will exhaust their budget. $r$ denotes the willingness to pay for the ideal differentiated good at location $\omega$.

I call the model a location model if the consumer heterogeneity over locations does not affect their preferences but leads to different transportation costs which have to be paid out of their budget. Hence, the transportation cost increases the price a consumer effectively has to pay to $p_{i}+t\left(\left|\omega-l_{i}\right|\right)$. The direct conditional utility functions and 
budget constraints in a location model are given by

$$
\begin{aligned}
\tilde{u}_{\mathbf{0}}^{\omega}\left(x_{\mathbf{0}}\right)=x_{\mathbf{0}} ; & y \geq x_{\mathbf{0}} \\
\tilde{u}_{i}^{\omega}\left(x_{\mathbf{0}}\right)=r+x_{\mathbf{0}} ; & y \geq x_{\mathbf{0}}+p_{i}+t\left(\left|\omega-l_{i}\right|\right), i=1, \ldots, n
\end{aligned}
$$

According to the specification goods are perfect substitutes and consumers have identical utility functions. A heterogeneity of consumers can only arise because their transportation cost enters their budget constraints.

In models of product differentiation the transportation cost has to be interpreted as a disutility in the utility function. All consumers pay the same price but their preferences on the differentiated goods are different. According to this terminology a spatial model in which the transportation cost is travel time and thus a disutility, is analyzed as a model of product differentiation. If a consumer chooses option $i$ her budget constraint is denoted by $\left(b c_{i}\right)$.

$$
\begin{aligned}
u_{0}^{\omega}\left(x_{0}\right)=x_{0} ; \quad\left(b c_{0}\right) & : y \geq x_{0} \\
u_{i}^{\omega}\left(x_{0}\right)=r-t\left(\left|\omega-l_{i}\right|\right)+x_{0} ; \quad\left(b c_{i}\right): & \geq x_{0}+p_{i}, i=1, \ldots, n
\end{aligned}
$$

When in a spatial model only part of the transportation cost is to be thought of a disutility then the consumer decision problem has to be formulated using a convex combination of (2) and (3). The nonexistence problem of the next section also holds for this case.

Up to a constant, (3) and (1) give the same 'utility' values when consumers can afford to buy the goods.

Utility maximization can be written as $\max \left\{\max _{x_{0}} u_{0}\left(x_{\mathbf{0}}\right)\right.$ s.t. $\left(b c_{0}\right),\left(\max _{x_{0}} u_{i}\left(x_{\mathbf{0}}\right)\right.$ s.t. $\left.\left.\left(b c_{i}\right)\right)_{i}\right\}$. The individual demand function for good $i=1, \ldots, n$ following from (3) takes values

$$
\xi_{i}^{\omega}(p, y)=\left\{\begin{array}{l}
1 \quad \text { if } p_{i} \leq y \text { and } p_{i} \leq r-t\left(\left|\omega-l_{i}\right|\right) \\
\quad \text { and } p_{i}-p_{j} \leq t\left(\left|\omega-l_{j}\right|\right)-t\left(\left|\omega-l_{i}\right|\right) \text { for all } j \neq i \text { with } p_{j}<y \\
0 \quad \text { if } p_{i}>y \text { or } p_{i}>r-t\left(\left|\omega-l_{i}\right|\right) \\
\quad \text { or } p_{i}-p_{j}>t\left(\left|\omega-l_{j}\right|\right)-t\left(\left|\omega-l_{i}\right|\right) \text { for some } j \neq i \text { with } p_{j}<y
\end{array}\right.
$$

An option is not feasible for a particular consumer if the price of one unit exceeds his capacity to pay. A consumer buys one unit of good $i$ if she can afford to buy it, if she prefers it to the outside option, and if she prefers it to all other feasible options in which she buys one unit of some other good $j \neq i$. 
Remark 1 The functions with values $v^{\omega, y}(p)$ and $z=r$ are indirect utility functions (for all $\omega$ ) if $r \leq y$.

This remark is proved as follows. First I show that (1) is an indirect utility function to (2). When a consumer cannot afford to buy good $i$ she also would not like to buy it compared to the outside option. Then one can replace $x_{0}$ by $y-p_{i}-t\left(\left|\omega-l_{i}\right|\right)$ in the conditional utility function for good $i$ and $y$ by $x_{0}$ for good 0 . Rescaling all conditional utility functions by subtracting $y$ gives (1) with $z=r$.

Since $y \geq r \geq r-t\left(\left|\omega-l_{i}\right|\right)$ a consumer who cannot buy good $i$ also does not want to do so in the model of product differentiation. Replacing $x_{0}$ by $y-p_{i}$ for good $i$ and by $y$ for good 0 and rescaling as above gives (1) with $z=r$.

When $r=y$, the maximal budget $y$ available for the differentiated market coincides with the maximal amount a consumer would be willing to give up to buy a good at his ideal point. When $r>y$, evaluation function (1) with the choice $z=y$ is an indirect utility function in the location model.

Without a restriction on $y$ relative to $r$ the evaluation function defined in (1) cannot be used in models of horizontal product differentiation: $v^{\omega}$ is not an indirect utility function because a consumer cannot afford to buy a good at a sufficiently high price.

Remark 2 In a model of product differentiation an evaluation function specified by (1) is inconsistent with utility maximization under a budget constraint.

The proof goes as follows: for $p_{i} \leq y,(3)$ is determined (up to monotone transformations) by (1). Hence it is sufficient to show that (1) generates demand functions which differ from (4) if $r>y$. For prices $y<p_{n}<r$ a consumer would rather buy his ideal good with $l_{i}=\omega$ than the outside option but she cannot afford to do so. There is no choice for $z$ in (1) which generates (4).

\section{Horizontal product differentiation with Hetero- geneous Income}

In this section I first explain why there cannot exist symmetric price equilibria in unit demand models with heterogeneous preferences and sufficiently heterogeneous income. 


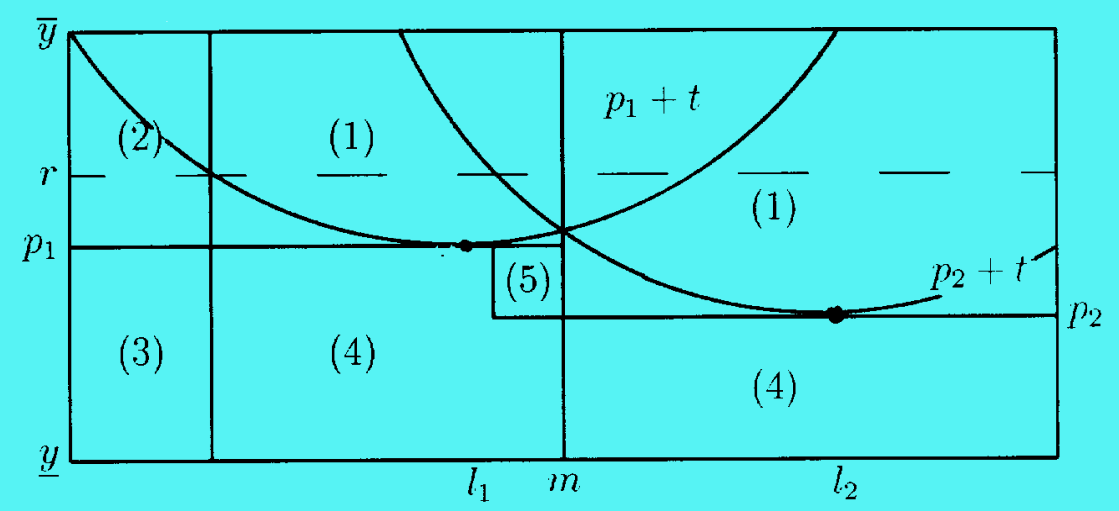

Figure 1: Consumers in a Model of Product Differentiation.

Figure 1:

Then I show nonexistence of equilibrium in a particular model. For simplicity, firms are assumed to have constant marginal costs of production $c$ (this can be easily extended). Hence firms maximize profits $\left(p_{i}-c\right) X_{i}(p)$ with respect to $p_{i}$ where $X_{i}$ is the demand of firm $i$. Consider a model of product differentiation in which all consumers are identical apart from their ideal point $\omega$ and their income $y$. A consumer type is identified by a point in the $(\omega, y)$-space (see Figure 1).

Proposition 1 For locations $l_{i} \neq l_{j}$ for all $j \neq i$, if $(A)$ holds there does not exist an imperfectly competitive equilibrium in which $p_{1}=p_{i}, i=2 \ldots n$.

Sketch of Proof. In any equilibrium $p_{i}>c$ and $p_{i}<\bar{y}$ for all $i=1, \ldots, n$. Isoprofit curves of firm $i$ are smooth in the $\left(p_{i}, X_{i}\right)$-space. Because of $(\mathrm{A})$ an inside kink of the demand curve occurs at $p_{1}=p_{i}>c$ for all $i=2, \ldots, n$. Due to the property of this kink, profits locally improve by a price change above or below $p_{i}=p_{j}, j \neq i$, or reach a local minimum in $p_{i}$ at $p_{i}=p_{j}, j \neq i$ for any $p_{j}$ with $c<p_{j}<\bar{y}$. 
The result is easily extended to transportation cost functions which partly operate on the utility function and partly are a real cost in the budget. Also a heterogeneous willingness to pay $r$ can be incorporated (under some restrictions on the stochastic dependence of $r$ and $y$ ). On the robustness of the result see also the concluding remarks.

I will demonstrate the importance of the kink of the demand functions in a particular specification. I take Hotelling's duopoly model with quadratic disutilities in distance (d'Aspremont, Gabszewicz, and Thisse, 1979) supplemented by consumers who are uniformly distributed by income. In particular, let $\omega$ be uniformly distributed on $[0,1]$ and $y \mid \omega$ be uniformly distributed on $[0,1]$ for all $\omega \in[0,1]$. The disutility in distance takes values $t(d)=\tau d^{2}, \tau>0$. Without loss of generality the mass of consumers is 1 . Aggregate demand for good 1 with $l_{1}<l_{2}$ (excluding consumers in situation (5)) is given by the following expression

$$
\hat{X}_{1}(p)=\left(1-p_{1}\right)\left(\min \left\{\frac{p_{2}-p_{1}}{2 \tau\left(l_{2}-l_{1}\right)}+\frac{l_{1}+l_{2}}{2}, l_{1}+\sqrt{\frac{r-p_{1}}{\tau}}\right\}-\max \left\{0, l_{1}-\sqrt{\frac{r-p_{1}}{\tau}}\right\}\right)
$$

for $p_{1} \leq 1$ and 0 for $p_{1}>1$. Analogously for good 2. $\hat{X}_{1}$ would arise in an ad hoc model in which all consumers have identical income $y \geq r$ and linear demand $1-p_{i}$. In order to reduce the number of intervals to be considered for the demand functions, I assume that $r$ is sufficiently high such that there are no consumers in situations (2) and (3). I now write aggregate demand functions $X_{i}, i=1,2$, as the mass of consumers who are in situations (1) or (5) who buy good $i$, i.e. $X_{i}=\hat{X}_{i}+\check{X}_{i}$. One has

$$
\begin{aligned}
& \hat{X}_{1}(p)=\left(1-p_{1}\right)\left(\frac{p_{2}-p_{1}}{2 \tau\left(l_{2}-l_{1}\right)}+\frac{l_{1}+l_{2}}{2}\right) \\
& \hat{X}_{2}(p)=\left(1-p_{2}\right)\left(1-\frac{p_{2}-p_{1}}{2 \tau\left(l_{2}-l_{1}\right)}-\frac{l_{1}+l_{2}}{2}\right) \\
& \check{X}_{1}(p)= \begin{cases}\left(p_{2}-p_{1}\right)\left(1-\frac{p_{2}-p_{1}}{2 \tau\left(l_{2}-l_{1}\right)}-\frac{l_{1}+l_{2}}{2}\right) & \text { if } p_{1}<p_{2} \\
0 & \text { if } p_{1} \geq p_{2}\end{cases} \\
& \check{X}_{2}(p)= \begin{cases}\left(p_{1}-p_{2}\right)\left(\frac{p_{2}-p_{1}}{2 \tau\left(l_{2}-l_{1}\right)}+\frac{l_{1}+l_{2}}{2}\right) & \text { if } p_{2}<p_{1} \\
0 & \text { if } p_{2} \geq p_{1}\end{cases}
\end{aligned}
$$

$X_{1}$ has a kink in $p_{1}$ at $p_{1}^{k}=p_{2}$. At $p_{1}^{k}+\epsilon, \epsilon>0$ the demand function is flatter than at $p_{1}^{k}-\epsilon$ because

$$
\lim _{p_{1} / p_{2}} \frac{\partial \breve{X}_{1}}{\partial p_{1}}(p)<0
$$


Analogously for good 2. The two goods are produced by two single-product firms at constant marginal cost of production $c$. Contrary to the model with identical income (and contrary to the location model, see concluding remarks) there cannot exist a symmetric equilibrium in prices. In particular, the result holds for symmetric locations. However, there may be asymmetric price equilibria.

I will restrict attention to the case of symmetric locations, i.e. $l_{2}=1-l_{1}$. If there is an equilibrium $p_{1}<p_{2}$ or $p_{1}>p_{2}$. Because of the symmetry of the model asymmetric equilibria come in pairs. In order to know the set of equilibria it is sufficient to look for equilibrium prices $p_{1}>p_{2}$ for given locations. In such an equilibrium first-order conditions of profit maximization are

$$
\begin{aligned}
& \left(-2 p_{1}+1+c\right)\left(p_{2}-p_{1}+\tau\left(1-2 l_{1}\right)\right)=-p_{1}^{2}+(1+c) p_{1}-c \\
& p_{2}=\frac{p_{1}+c}{2}+\frac{\frac{3}{2}\left(1-p_{1}\right)}{1+\frac{1-p_{1}}{2 \tau\left(1-2 l_{1}\right)}}
\end{aligned}
$$

Substituting (6) into (5) gives a polynomial of degree 3 for which an explicit solution can be obtained. I will impose particular values for the parameters and analyze whether under these parameters an equilibrium does exist.

Consider the parameter constellation $c=0, \tau=1$ under maximal differentiation, i.e. $l_{1}=0, l_{2}=1$.

Proposition 2 For $c=0, \tau=1, l_{1}=0, l_{2}=1$, there does not exist a price equilibrium in pure strategies.

Proof. In an equilibrium first-order conditions (5) and (6) have to be satisfied. Under the parameter choice one obtains $p_{1}=0.417$ as the only admissible solution to the polynomial of degree 3 of (5) where $p_{2}$ is replaced by the right-hand side of (6). It follows that $p_{2}=0.886$ which contradicts the assumption that $p_{1}>p_{2}$ under which the equation system was derived.

The result holds for any symmetric location and for other parameter choices where $\tau>0$ and $c$ are free to choose. By continuity the result holds in a neighborhood around symmetric locations. Hence, existence of price equilibrium is not a generic property in the parameter space $[0,1] \times[0,1] \times \Re_{+}^{2}$. The nonexistence implies that there does not exist a subgame perfect equilibrium of the product choice-then-price game. 


\section{An example with unit-elastic demand}

In this section I present a different specification of a Hotelling model in which consumers have unit elastic demand. With this specification one avoids the problem of the kinked demand which occurs with unit demand or, more general, quasi-linear preferences. Further more elaborate examples are presented in Peitz (1998). In other work I generalize this specification to a wider set of individual demand functions. As before, $l_{i} \in[0,1]$ denotes the location of the good in the product space, $l \equiv\left(l_{i}\right)_{i}$, and $\Omega=[0,1]$ is the space of taste parameters of the consumers which determines the relative evaluation of the differentiated goods. Taste parameter $\alpha \in A \subseteq[0,1]$ determines the relative evaluation of any differentiated good relative to the composite commodity. The utility function of a consumer with taste parameters $(\omega, \alpha) \in \Omega \times A$ is defined as

$$
u\left(x_{\mathbf{0}}, x, l\right)=\left(\sum_{i} \tilde{u}_{i}\left(x_{i}, l_{i}\right)\right)^{\alpha} x_{\mathbf{0}}^{1-\alpha}
$$

where $\tilde{u}_{i}\left(x_{i}, l_{i}\right)$ denotes the direct conditional utility function. If $\tilde{u}_{i}\left(x_{i}, l_{i}\right)$ is convex in $x_{i}$, a consumer buys only one good in the differentiated market. The conditional utility function takes the form

$$
\tilde{u}_{i}\left(x_{i}, l_{i}\right)=x_{i} e^{-\left(\omega-l_{i}\right)^{2}} .
$$

Hence, the consumer spends $\alpha y$ in the differentiated market. If a consumer buys good $i$, then a monotone transformation of indirect utility is $v_{i}^{\omega, y}\left(p_{i}\right)=\frac{1}{\alpha} y-\log p_{i}-$ $\left(\omega-l_{i}\right)^{2}$. Consequently, in a duopoly a consumer has the demand function

$$
\xi_{i}^{\omega}(p, y)= \begin{cases}\alpha y & \text { if } \log p_{1}-\log p_{2}<2 \omega\left(l_{1}-l_{2}\right)+l_{2}^{2}-l_{1}^{2} \\ 0 & \text { else }\end{cases}
$$

The decision to change from one good to the other has the same formula as in the Hotelling model with quadratic transportation costs except that price differences are replaced by logarithmic price differences.

Consider a population which is heterogeneous in income and taste parameter $\omega$. Consider the example in which $y$ and $\omega$ are stochastically independent and $\omega$ is uniformly distributed on $[0,1]$. In a duopoly demand for firm $1, l_{1}<l_{2}$, is $X_{1}(p)=\frac{a m \bar{y}}{p_{1}}$ where $\bar{y}$ is mean income and $m$ is the taste parameter of the marginal consumers who are indifferent between the two goods. In this case it is easy to show the existence of a unique price equilibrium (see e.g. Peitz, 1997). In addition, prices for symmetric locations can be calculated. 
Proposition 3 For symmetric locations $\left(l_{1}, 1-l_{1}\right), l_{1} \leq \frac{1}{2}$, equilibrium prices are $p_{1}^{*}=p_{2}^{*}=c\left(2-2 l_{1}\right)$.

Proof. Define $\tilde{m}: \Re \rightarrow \Re$ with $\tilde{m}(\theta)=\frac{\theta}{2\left(l_{2}-l_{1}\right)}+\frac{l_{1}+l_{2}}{2}$ where $\theta \equiv \log p_{2}-\log p_{1}$. The first-order conditions of profit maximization then can be written as

$$
\begin{aligned}
c \tilde{m}(\theta) & =\left(p_{1}-c\right) \tilde{m}^{\prime}(\theta) \\
c(1-\tilde{m}(\theta)) & =\left(p_{2}-c\right) \tilde{m}^{\prime}(\theta)
\end{aligned}
$$

Rearranging and taking ratios yields

$$
\frac{1-\tilde{m}(\theta)+\frac{1}{2\left(l_{2}-l_{1}\right)}}{\tilde{m}(\theta)+\frac{1}{2\left(l_{2}-l_{1}\right)}}-e^{\theta}=0 .
$$

Clearly, $\theta=0$ is a solution for $l_{2}=1-l_{1}$. One can show that the left-hand side is a decreasing function in $\theta$. Hence, $\theta=0$ is the unique zero and $p_{1}^{*}=p_{2}^{*}$. Substituting $p_{1}=p_{2}$ and $l_{2}=1-l_{1}$ into the first of the first-order conditions gives $p_{1}^{*}=c\left(2-2 l_{1}\right)$.

From the explicit formula of equilibrium prices it follows that the markups $\frac{p_{i}-c}{c}$ are constant in marginal costs. This is different from the Hotelling model with quadratic transportation costs where the price-cost margin $p_{i}-c$ is constant. For more details see Peitz (1998).

\section{Concluding Remarks}

In this paper I provided two insights: first, location models and models of product differentiation are formally not equivalent. Second, in models of product differentiation there cannot exist symmetric price equilibria if some consumers are sufficiently poor. Furthermore, in a standard specification also asymmetric price equilibria fail to exist and consequently there does not exist a subgame perfect equilibrium of the product choice-then-price game when some consumers are constrained due to the budget constraint. The inside kinks in the aggregate demand functions are independent of the particular specification of the "transportation cost function".

A model in which transportation costs are real costs this problem does not arise: individual and aggregate demand functions in the associated location model differ from the model of product differentiation and there exists a subgame perfect equilibrium for the specification which has been considered. The model with quadratic transportation costs is merely a reformulation of Böckem (1994). Böckem (1994, 1996) shows 
the uniqueness of price equilibrium when reservation prices (not income) are heterogeneous.

The existence problem in the model of product differentiation is due to quasi-linear preferences of the consumers. I took a specification of horizontal product differentiation and unit demand. Kinks in the aggregate demand functions also occur in models with random utility and unit demand. Also in models of vertical product differentiation an inside kink occurs if income and quality sensitivity of the consumers do not depend in a particular way on each other. The model of Gabszewicz and Thisse (1979) is such an exception. Whether or not an equilibrium exists in a model of vertical product differentiation with inside kinks depends on the specification and the parameter choices. Clearly, from Cremer and Thisse (1991) it is known that one can rewrite a horizontal model as a vertical model of product differentiation and hence one can obtain nonexistence of equilibrium. The nonexistence problem (i.e., inside kink) seems to be a general property of unit demand models with heterogeneous preferences and sufficiently heterogeneous income. It is an open question whether one can find a formulation of unit demand which is consistent with some consumer theory and which does not lead to existence problems of the kind pointed out in this paper.

Finally, not only models of product differentiation suffer from the problem pointed out in this paper. In models of search or switching costs, when the search cost or switching cost respectively is not a monetary cost but a disutility one encounters the same type of problems whenever one has unit demand, and heterogeneous search or switching costs, and income heterogeneity. (On the literature on switching costs see e.g. Klemperer, 1995). 


\section{References}

[1] Anderson, S., A. de Palma, and J. Thisse (1992), Discrete Choice Theory of Product Differentiation, Cambridge, MA: MIT Press.

[2] Beath, J. and Y. Katsoulacos (1991), The Economic Theory of Product Differentiation, Cambridge: Cambridge University Press.

[3] Böckem, S. (1994), A Generalized Model of Horizontal Product Differentiation, Journal of Industrial Economics 42, 287-298.

[4] Böckem, S. (1996), Corrigendum to: A Generalized Model of Horizontal Product Differentiation, mimeo.

[5] Caplin, A. and B. Nalebuff (1991), Aggregation and Imperfect Competition: on the Existence of Equilibrium, Econometrica 59, 25-59.

[6] Cremer, H. and J.-F. Thisse (1991), Location Models of Horizontal Differentiation: A Special Case of Vertical Differentiation, Journal of Industrial Economics 39, 383-390.

[7] D'Aspremont, C., J.J. Gabszewicz, and J.F. Thisse (1979), On Hotelling's 'Stability in Competition', Econometrica 47, 1145-1150.

[8] Economides, N. (1984), The Principle of Minimum Differentiation Revisited, European Economic Review 24, 345-368.

[9] Economides, N. (1989), Symmetric Equilibrium Existence and Optimality in a Differentiated Product Market, Journal of Economic Theory 47, 178-194.

[10] Gabszewicz, J.J. and J.-F. Thisse (1979), Price Competition, Quality and Income Disparities, Journal of Economic Theory 20, 340-359.

[11] Hotelling, H. (1929), Stability in Competition, Economic Journal 39, 41-57.

[12] Klemperer, P. (1995), Competition when Consumers have Switching Costs: An Overview with Applications to Industrial Organization, Macroeconomics, and International Trade, Review of Economic Studies 62, 515-540.

[13] Mussa, M. and S. Rosen (1978), Monopoly and Product Quality, Journal of Economic Theory 18, 301-317. 
[14] Peitz, M. (1995), Utility Maximization in Models of Discrete Choice, Economics Letters 49, 91-94.

[15] Peitz, M. (1996), The Circular Road Revisited, mimeo.

[16] Peitz, M. (1997), Differentiated Bertrand Duopoly with Variable Demand, Research in Economics/Ricerche Economiche 51, 85-100.

[17] Peitz, M. (1998), Two-Stage Models of Product Differentiation with Unit-Elastic Demand, mimeo.

[18] Salop, S. (1979), Monopolistic Competition with Outside Goods, Bell Journal of Economics 10, 141-156. 